\title{
A Local Non-Abelian Gauge Invariant Action Stemming from the Nonlocal Operator $F_{\mu v}\left(D^{2}\right)^{-1} F_{\mu v}$
}

\author{
D. Dudal ${ }^{a},{ }^{*}$ M. A. L. Capri ${ }^{b}$, J. A. Gracey ${ }^{c}$, V. E. R. Lemes ${ }^{b}$, R. F. Sobreiro ${ }^{b}$, S. P. Sorella ${ }^{b},^{\dagger}$ and H. Verschelde ${ }^{a}$ \\ a Ghent University \\ Department of Mathematical Physics and Astronomy, \\ Krijgslaan 281-S9, B-9000 Gent, Belgium \\ ${ }^{b}$ UERJ - Universidade do Estado do Rio de Janeiro, \\ Rua São Francisco Xavier 524, \\ 20550-013 Maracanã, Rio de Janeiro, Brazil
}

\author{
${ }^{c}$ Theoretical Physics Division, Department of Mathematical Sciences, \\ University of Liverpool, P.O. Box 147, Liverpool, L69 3BX, United Kingdom
}

(Received on 12 September, 2006)

\begin{abstract}
We report on the nonlocal gauge invariant operator of dimension two, $F_{\mu v}\left(D^{2}\right)^{-1} F_{\mu v}$. We are able to localize this operator by introducing a suitable set of (anti)commuting antisymmetric tensor fields. Starting from this, we succeed in constructing a local gauge invariant action containing a mass parameter, and we prove the renormalizability to all orders of perturbation theory of this action in the linear covariant gauges using the algebraic renormalization technique. We point out the existence of a nilpotent BRST symmetry. Despite the additional (anti)commuting tensor fields and coupling constants, we prove that our model in the limit of vanishing mass is equivalent with ordinary massless Yang-Mills theories by making use of an extra symmetry in the massless case. We also present explicit renormalization group functions at two loop order in the $\overline{\mathrm{MS}}$ scheme.
\end{abstract}

Keywords: Yang-Mills gauge theory; BRST symmetry; Renormalization; Mass

\section{INTRODUCTION}

We shall consider pure Euclidean $S U(N)$ Yang-Mills theories with action

$$
S_{Y M}=\frac{1}{4} \int d^{4} x F_{\mu v}^{a} F_{\mu v}^{a}
$$

where $A_{\mu}^{a}, a=1, \ldots, N^{2}-1$ is the gauge boson field, with associated field strength

$$
F_{\mu \nu}^{a}=\partial_{\mu} A_{v}^{a}-\partial_{v} A_{\mu}^{a}+g f^{a b c} A_{\mu}^{b} A_{v}^{c} .
$$

The theory (1) is invariant with respect to the local gauge transformations

$$
\delta A_{\mu}^{a}=D_{\mu}^{a b} \omega^{b}
$$

with

$$
D_{\mu}^{a b}=\partial_{\mu} \delta^{a b}-g f^{a b c} A_{\mu}^{c},
$$

denoting the adjoint covariant derivative.

As it is well known, the theory (1) is asymptotically free $[1,2]$, i.e. the coupling becomes smaller at higher energies and vice versa. At very high energies, the interaction is weak and the gluons can be considered as almost free particles.

\footnotetext{
* Postdoctoral fellow of the Special Research Fund of Ghent University. †Work supported by FAPERJ, Fundação de Amparo à Pesquisa do Estado do Rio de Janeiro, under the program Cientista do Nosso Estado, E26/151.947/2004.
}

However, in spite of the progress in the last decades, we still lack a satisfactory understanding of the behaviour of YangMills theories in the low energy regime. Here the coupling constant of the theory is large and nonperturbative effects have to be taken into account.

The introduction of condensates, i.e. the (integrated) vacuum expectation value of certain operators, allows one to parametrize certain nonperturbative effects arising from the infrared sector of e.g. the theory described by (1). Via the Operator Product Expansion (OPE) (viz. short distance expansion), which is applicable to local operators, one can relate these condensates to power corrections which give nonperturbative information in addition to the perturbatively calculable results. If one wants to consider the possible effects of condensates on physical quantities in a gauge theory, quite clearly only gauge invariant operators should be considered. The most famous example is the dimension 4 gluon condensate $\left\langle\alpha_{s} F_{\mu \nu}^{2}\right\rangle$, giving rise to $\frac{1}{Q^{4}}$ power corrections. Via the SVZ (Shifman-Vainshtein-Zakharov) sum rules [3], one can extract phenomenological estimates for $\left\langle\alpha_{s} F_{\mu v}^{2}\right\rangle$.

In recent years, a great deal of interest arose in dimension 2 condensates in gauge theories. Most attention was paid to the gluon condensate $\left\langle A_{\mu}^{2}\right\rangle$ in the Landau gauge, due to the work of $[4,5]$, as the quantity

$$
\left\langle A_{\min }^{2}\right\rangle \equiv \min _{U \in S U(N)} \frac{1}{V T} \int d^{4} x\left\langle\left(A_{\mu}^{U}\right)^{2}\right\rangle
$$

which is gauge invariant due to the minimization along the gauge orbits, could be physically relevant. In fact, as shown in $[4,5]$ in the case of compact QED, the quantity $\left\langle A_{\min }^{2}\right\rangle$ seems to be useful in order to detect the presence of nontrivial field configurations like monopoles. One can show that $A_{\mathrm{min}}^{2}$ 


$$
A_{\min }^{2}=\int d^{4} x\left[A_{\mu}^{a}\left(\delta_{\mu v}-\frac{\partial_{\mu} \partial_{v}}{\partial^{2}}\right) A_{v}^{a}-g f^{a b c}\left(\frac{\partial_{v}}{\partial^{2}} \partial A^{a}\right)\left(\frac{1}{\partial^{2}} \partial A^{b}\right) A_{v}^{c}\right]+O\left(A^{4}\right)
$$

Since the operator $A_{\min }^{2}$ is nonlocal, it falls beyond the applicability of the OPE annex sum rules, which refer to local operators.

However, in the Landau gauge, $\partial_{\mu} A_{\mu}=0$, all nonlocal terms of expression (6) drop out, so that $A_{\mathrm{min}}^{2}$ reduces to the local operator $A_{\mu}^{2}$, hence the interest in the Landau gauge and its dimension two gluon condensate $\left\langle A_{\mu}^{2}\right\rangle$. A complication is that the explicit determination of the absolute minimum of $A_{\mu}^{2}$ along its gauge orbit, and moreover of its vacuum expectation value, is a very delicate issue intimately related to the problem of Gribov copies [8-13].

Nevertheless, some nontrivial results were proven concerning the operator $A_{\mu}^{2}$. In particular, we mention its multiplicative renormalizability to all orders of perturbation theory, in addition to an interesting and numerically verified relation concerning its anomalous dimension $[14,15]$. An effective potential approach consistent with renormalizability and renormalization group requirements for local composite operators (LCO) has also been worked out for this operator, giving further evidence of a nonvanishing condensate $\left\langle A_{\mu}^{2}\right\rangle \neq 0$, which lowers the nonperturbative vacuum energy [16]. The LCO method yields an effective gluon mass squared $m_{g}^{2} \sim\left\langle A_{\mu}^{2}\right\rangle$ of a few hundred MeV [16-19].

In [20], it was already argued that gauge (in)variant condensates could also influence gauge variant quantities such as the gluon propagator. An OPE argument based on lattice simulations in the Landau gauge has indeed provided evidence that a condensate $\left\langle A_{\mu}^{2}\right\rangle$ could account for quadratic power corrections of the form $\sim \frac{1}{Q^{2}}$, reported in the running of the coupling constant as well as in the gluon propagator, see e.g. [21-26]. This OPE approach allows one to obtain an estimate for the soft part $\left\langle A_{\mu}^{2}\right\rangle_{I R}$ originating from the infrared sector. The OPE can also be employed to relate this condensate to an effective gluon mass [21].

The presence of mass parameters in the gluon propagator have also been advocated from the lattice perspective several times [27-32], whilst effective gluon masses also found phenomenological use [33, 34].

A somewhat weak point about the operator $A_{\min }^{2}$ is that it is unclear how to deal with it in gauges other than the Landau gauge. Till now, it seems hopeless to prove its renormalizability out of the Landau gauge. In fact, at the classical level, adding (5) to the Yang-Mills action is equivalent to add the so-called Stueckelberg action, which is known to be not renormalizable [35, 36]. We refer to [7] for details and references. As already mentioned, also the OPE becomes useless outside the Landau gauge for this particular operator.
In other gauges, there can be found other renormalizable local operators, which condense and give rise to a dynamical gluon mass. Next to the Landau gauge [14, 16-19, 37, 38] the maximal Abelian [39, 40], linear covariant [41-43] and CurciFerrari gauges $[44,45]$ have been investigated in the past.

The relevant operators in these other gauges are however gauge variant, and as a consequence also the effective gluon mass. From this perspective, it is worthwhile to find out whether a gauge invariant framework might be found for a dynamical mass, and related to it for $\frac{1}{Q^{2}}$ power corrections.

In order to have a starting point, we need a dimension 2 operator that is gauge invariant. This necessarily implies a nonlocal operator, since gauge invariant local operators of dimension 2 do not exist. We would also need a consistent calculational framework, which requires an action only containing local terms. Therefore, we should find an operator that can be localized by means of a finite set of auxiliary fields, in such a way that the local gauge invariance is respected. As $A_{\text {min }}^{2}$ looks a bit hopeless from this viewpoint as it is a infinite series of nonlocal terms (6), we moved our attention instead to the nonlocal gauge invariant operator

$$
O \equiv \frac{1}{V T} \int d^{4} x F_{\mu \nu}^{a}\left[\left(D^{2}\right)^{-1}\right]^{a b} F_{\mu \nu}^{b}
$$

This operator caught already some attention in 3 dimensional gauge theories in relation to a dynamical mass generation [46].

In the following sections, we shall show that the operator (7) can be localized, giving rise to a local, classically gauge invariant action. Afterwards, we discuss how to investigate the renormalizability of the action once quantized. Eventually, we need to introduce a slightly more general classical action in order to obtain a quantum action that is renormalizable to all orders of perturbation theory. In the case of vanishing mass, the equivalence of our action with usual Yang-Mills theories can be shown. We shall point out the existence of a naturally extended version of the usual BRST symmetry. Before turning to conclusions, we explicitly give various renormalization group functions, verifying the renormalizability at the practical level. 


\section{CONSTRUCTION OF THE ACTION AND ITS RENORMALIZABILITY AT THE QUANTUM LEVEL}

\section{A. The action at the classical level}

We can add the operator (7) to the Yang-Mills action as a mass term via

$$
S_{Y M}+S_{O}
$$

with

$$
S_{O}=-\frac{m^{2}}{4} \int d^{4} x F_{\mu v}^{a}\left[\left(D^{2}\right)^{-1}\right]^{a b} F_{\mu v}^{b} .
$$

As we have discussed in [7], the action (8) can be localized by introducing a pair of complex bosonic antisymmetric tensor fields, $\left(B_{\mu \nu}^{a}, \bar{B}_{\mu \nu}^{a}\right)$, and a pair of complex anticommuting antisymmetric tensor fields, $\left(\bar{G}_{\mu \nu}^{a}, G_{\mu \nu}^{a}\right)$, belonging to the adjoint representation, according to which

$$
\begin{aligned}
e^{-S_{O}} & =\int D \bar{B} D B D \bar{G} D G \exp \left[-\left(\frac{1}{4} \int d^{4} x \bar{B}_{\mu \nu}^{a} D_{\sigma}^{a b} D_{\sigma}^{b c} B_{\mu \nu}^{c}\right.\right. \\
& \left.\left.-\frac{1}{4} \int d^{4} x \bar{G}_{\mu \nu}^{a} D_{\sigma}^{a b} D_{\sigma}^{b c} G_{\mu \nu}^{c}+\frac{i m}{4} \int d^{4} x(B-\bar{B})_{\mu \nu}^{a} F_{\mu \nu}^{a}\right)\right]
\end{aligned}
$$

Therefore, we obtain a classical local action which reads

$$
S_{Y M}+S_{B G}+S_{m}
$$

where

$$
\begin{aligned}
S_{B G} & =\frac{1}{4} \int d^{4} x\left(\bar{B}_{\mu \nu}^{a} D_{\sigma}^{a b} D_{\sigma}^{b c} B_{\mu \nu}^{c}-\bar{G}_{\mu v}^{a} D_{\sigma}^{a b} D_{\sigma}^{b c} G_{\mu \nu}^{c}\right), \\
S_{m} & =\frac{i m}{4} \int d^{4} x(B-\bar{B})_{\mu \nu}^{a} F_{\mu \nu}^{a},
\end{aligned}
$$

which is left invariant by the gauge transformations

$$
\begin{aligned}
\delta A_{\mu}^{a} & =-D_{\mu}^{a b} \omega^{b}, \\
\delta B_{\mu \nu}^{a} & =g f^{a b c} \omega^{b} B_{\mu \nu}^{c}, \delta \bar{B}_{\mu \nu}^{a}=g f^{a b c} \omega^{b} \bar{B}_{\mu \nu}^{c}, \\
\delta G_{\mu \nu}^{a} & =g f^{a b c} \omega^{b} G_{\mu \nu}^{c}, \delta \bar{G}_{\mu \nu}^{a}=g f^{a b c} \omega^{b} \bar{G}_{\mu \nu}^{c} .
\end{aligned}
$$

\section{B. The action at the quantum level}

In order to discuss the renormalizability of (11), we relied on a method introduced by Zwanziger in [47, 48]. Instead of using (11) with $m$ coupled to the composite operators $B_{\mu \nu}^{a} F_{\mu v}^{a}$ and $B_{\mu \nu}^{a} F_{\mu \nu}^{a}$, we introduce 2 suitable external sources $V_{\rho \sigma \mu \nu}$ and $\bar{V}_{\rho \sigma \mu \nu}$ and replace $S_{m}$ by

$$
\frac{1}{4} \int d^{4} x\left(V_{\sigma \rho \mu \nu} \bar{B}_{\sigma \rho}^{a} F_{\mu \nu}^{a}-\bar{V}_{\sigma \rho \mu \nu} B_{\sigma \rho}^{a} F_{\mu \nu}^{a}\right)
$$

At the end, the sources $V_{\sigma \rho \mu v}(x), \bar{V}_{\sigma \rho \mu v}(x)$ are required to attain their physical value, namely

$$
\left.\bar{V}_{\sigma \rho \mu \nu}\right|_{\text {phys }}=\left.V_{\sigma \rho \mu \nu}\right|_{\text {phys }}=-\frac{i m}{2}\left(\delta_{\sigma \mu} \delta_{\rho v}-\delta_{\sigma v} \delta_{\rho \mu}\right),
$$

so that (14) reduces to $S_{m}$ in the physical limit.

From now on, we assume the linear covariant gauge fixing, implemented through

$$
S_{g f}=\int d^{4} x\left(\frac{\alpha}{2} b^{a} b^{a}+b^{a} \partial_{\mu} A_{\mu}^{a}+\bar{c}^{a} \partial_{\mu} D_{\mu}^{a b} c^{b}\right),
$$

In [7], we wrote down a list of symmetries enjoyed by the action

$$
S_{Y M}+S_{B G}+S_{g f}
$$

i.e. in absence of the sources. Let us only mention here the BRST symmetry, generated by the nilpotent transformation $s$ given by

$$
\begin{aligned}
s A_{\mu}^{a} & =-D_{\mu}^{a b} c^{b}, s c^{a}=\frac{g}{2} f^{a b c} c^{a} c^{b}, \\
s B_{\mu \nu}^{a} & =g f^{a b c} c^{b} B_{\mu \nu}^{c}+G_{\mu \nu}^{a}, s \bar{B}_{\mu \nu}^{a}=g f^{a b c} c^{b} \bar{B}_{\mu \nu}^{c}, \\
s G_{\mu \nu}^{a} & =g f^{a b c} c^{b} G_{\mu \nu}^{c}, s \bar{G}_{\mu \nu}^{a}=g f^{a b c} c^{b} \bar{G}_{\mu \nu}^{c}+\bar{B}_{\mu \nu}^{a}, \\
s \bar{c}^{a} & =b^{a}, s b^{a}=0, s^{2}=0 .
\end{aligned}
$$

It turns out that one can introduce all the necessary external sources in a way consistent with the starting symmetries. This allows to write down several Ward identities by which the most general counterterm is restricted using the algebraic renormalization formalism [7, 49]. After a very cumbersome analysis, it turns out that the action (11) must be modified to

$$
S_{p h y s}=S_{c l}+S_{g f}
$$

with

$$
\begin{aligned}
S_{c l} & =\int d^{4} x\left[\frac{1}{4} F_{\mu \nu}^{a} F_{\mu \nu}^{a}+\frac{i m}{4}(B-\bar{B})_{\mu \nu}^{a} F_{\mu \nu}^{a}+\frac{1}{4}\left(\bar{B}_{\mu \nu}^{a} D_{\sigma}^{a b} D_{\sigma}^{b c} B_{\mu \nu}^{c}-\bar{G}_{\mu \nu}^{a} D_{\sigma}^{a b} D_{\sigma}^{b c} G_{\mu \nu}^{c}\right)\right. \\
& \left.-\frac{3}{8} m^{2} \lambda_{1}\left(\bar{B}_{\mu \nu}^{a} B_{\mu \nu}^{a}-\bar{G}_{\mu \nu}^{a} G_{\mu \nu}^{a}\right)+m^{2} \frac{\lambda_{3}}{32}\left(\bar{B}_{\mu \nu}^{a}-B_{\mu \nu}^{a}\right)^{2}+\frac{\lambda^{a b c d}}{16}\left(\bar{B}_{\mu \nu}^{a} B_{\mu \nu}^{b}-\bar{G}_{\mu \nu}^{a} G_{\mu \nu}^{b}\right)\left(\bar{B}_{\rho \sigma}^{c} B_{\rho \sigma}^{d}-\bar{G}_{\rho \sigma}^{c} G_{\rho \sigma}^{d}\right)\right],
\end{aligned}
$$


in order to have renormalizability to all orders of perturbation theory. We notice that we had to introduce a new invariant quartic tensor coupling $\lambda^{a b c d}$, subject to the generalized Jacobi identity

$$
f^{m a n} \lambda^{m b c d}+f^{m b n} \lambda^{a m c d}+f^{m c n} \lambda^{a b m d}+f^{m d n} \lambda^{a b c m}=0,
$$

and the symmetry constraints

$$
\begin{aligned}
& \lambda^{a b c d}=\lambda^{c d a b}, \\
& \lambda^{a b c d}=\lambda^{b a c d},
\end{aligned}
$$

as well as two new mass couplings $\lambda_{1}$ and $\lambda_{3}$. Without the new couplings, i.e. when $\lambda_{1} \equiv 0, \lambda_{3} \equiv 0, \lambda^{a b c d} \equiv 0$, the previous action would not be renormalizable. We refer to [7, 50] for all the details. We also notice that the novel fields $B_{\mu \nu}^{a}, \bar{B}_{\mu \nu}^{a}$, $G_{\mu \nu}^{a}$ and $\bar{G}_{\mu \nu}^{a}$ are no longer appearing at most quadratically. As it should be expected, the classical action $S_{c l}$ is still gauge invariant w.r.t. (13).

\section{FURTHER PROPERTIES OF THE ACTION}

\section{A. Existence of a BRST symmetry with a nilpotent charge}

The BRST transformation (18) no longer generates a symmetry of the action $S_{\text {phys }}$. However, we are able to define a natural generalization of the usual BRST symmetry that does constitute an invariance of the gauge fixed action (19). Indeed, after inspection, one shall find that

$$
\begin{aligned}
\widetilde{s} S_{\text {phys }} & =0, \\
\widetilde{s}^{2} & =0,
\end{aligned}
$$

with

$$
\begin{aligned}
\widetilde{s}_{\mu}^{a} & =-D_{\mu}^{a b} c^{b}, \widetilde{s c}^{a}=\frac{g}{2} f^{a b c} c^{a} c^{b}, \\
\widetilde{s} B_{\mu \nu}^{a} & =g f^{a b c} c^{b} B_{\mu \nu}^{c}, \widetilde{s} \bar{B}_{\mu \nu}^{a}=g f^{a b c} c^{b} \bar{B}_{\mu \nu}^{c}, \\
\widetilde{s} G_{\mu \nu}^{a} & =g f^{a b c} c^{b} G_{\mu \nu}^{c}, \widetilde{s}_{\mu \nu}^{a}=g f^{a b c} c^{b} \bar{G}_{\mu \nu}^{c}, \\
\widetilde{s} \bar{c}^{a} & =b^{a}, \widetilde{s} b^{a}=0 .
\end{aligned}
$$

Hence, the action $S_{\text {phys }}$ is invariant with respect to a nilpotent BRST transformation $\widetilde{s}$. We obtained thus a gauge field theory, described by the action $S_{\text {phys }}$, (19), containing a mass term, and which has the property of being renormalizable, while nevertheless a nilpotent BRST transformation expressing the gauge invariance after gauge fixing exists simultaneously. It is clear that $\widetilde{s}$ stands for the usual BRST transformation, well known from literature, on the original Yang-Mills fields, whereas the gauge fixing part $S_{g f}$ given in (20) can be written as a $\widetilde{s}$-variation, ensuring that the gauge invariant physical operators shall not depend on the choice of the gauge parameter [49].

\section{B. Existence of a "supersymmetry" when $m \equiv 0$}

We define a nilpotent (anti-commuting) transformation $\delta_{s}$ as

$$
\begin{aligned}
\delta_{s} B_{\mu \nu}^{a} & =G_{\mu \nu}^{a}, & & \delta_{s} G_{\mu \nu}^{a}=0, \\
\delta_{s} \bar{G}_{\mu \nu}^{a} & =\bar{B}_{\mu \nu}^{a}, & & \delta_{s} \bar{B}_{\mu \nu}^{a}=0, \\
\delta_{s} \text { (rest) } & =0 & &
\end{aligned}
$$

Then one easily verifies that (25) generates a "supersymmetry" of the action $S_{\text {phys }}^{m \equiv 0}$ since

$$
\delta_{s} S_{\text {phys }}^{m \equiv 0}=0
$$

with

$$
\delta_{s}^{2}=0
$$

Taking another look at the transformations $s$ and $\widetilde{s}$, respectively given by (18) and (24), one recognizes that

$$
\begin{aligned}
s & =\widetilde{s}+\delta_{s}, \\
\left\{\delta_{s}, \widetilde{s}\right\} & =0 .
\end{aligned}
$$

Since $\delta_{s}$ is a nilpotent operator, it possesses its own cohomology, which is easily identified with polynomials in the original Yang-Mills fields $\left\{A_{\mu}^{a}, b^{a}, c^{a}, \bar{c}^{a}\right\}$. The auxiliary tensor fields, $\left\{B_{\mu \nu}^{a}, \bar{B}_{\mu \nu}^{a}, G_{\mu \nu}^{a}, \bar{G}_{\mu \nu}^{a}\right\}$, do not belong to the cohomology of $\delta_{s}$, because they form pairs of doublets [49]. This fact can be brought to use in the following subsection.

\section{Equivalence with Yang-Mills gauge theory when $m \equiv 0$}

If the mass $m \equiv 0$, we would expect that the action (19) would be equivalent with the usual Yang-Mills gauge theory, since in the nonlocal formulation (9), we would have introduced "nothing". In the local renormalizable formulation (19), this would also be trivially true when $\lambda^{a b c d} \equiv 0$ as then we would only have added a -although quite complicatedunity to the Yang-Mills action. Unfortunately, since renormalization forbids setting $\lambda^{a b c d}=0$, we must find another argument to relate $S_{p h y s}^{m \equiv 0}$ to the usual Yang-Mills gauge theory. As proven in [50], the "supersymmetry" $\delta_{s}$ of (25) can be used to show that

$$
\left\langle G_{n}\left(x_{1}, \ldots, x_{n}\right)\right\rangle_{S_{Y M}+S_{g f}} \equiv\left\langle G_{n}\left(x_{1}, \ldots, x_{n}\right)\right\rangle_{S_{p h y s}^{m=0}}
$$

where

$$
G_{n}\left(x_{1}, \ldots, x_{n}\right)=A\left(x_{1}\right) \ldots A\left(x_{i}\right) \bar{c}\left(x_{i+1}\right) \ldots \bar{c}\left(x_{j}\right) c\left(x_{j+1}\right) \ldots c\left(x_{k}\right) b\left(x_{k+1}\right) \ldots b\left(x_{n}\right)
$$


is a generic Yang-Mills functional. The expectation value of any Yang-Mills Green function, constructed from the fields $\left\{A_{\mu}^{a}, c^{a}, \bar{c}^{a}, b^{a}\right\}$ and calculated with the original (gauge fixed) Yang-Mills action $S_{Y M}+S_{g f}$, is thus identical to the one calculated with the massless action $S_{p h y s}^{m \equiv 0}$, where it is of course assumed that the gauge freedom of both actions has been fixed by an identical gauge fixing.

The foregoing result also reflects on the renormalization group functions. As usual, we employ a massless renormalization scheme known as the $\overline{\mathrm{MS}}$ scheme. As a consequence, we can set $m=0$ in order to extract the ultraviolet behaviour. Using (29), we conclude that all the renormalization group functions of the original Yang-Mills quantities are not affected by the presence of the extra fields or couplings. This fact shall be explicitly verified in the next section.

\section{EXPLICIT RENORMALIZATION AT TWO LOOP ORDER}

Having proven the renormalizability of the action (19), we shall now compute explicitly the two loop anomalous dimension of the fields and the one loop $\beta$-function of the tensor coupling $\lambda^{a b c d}$. The corresponding details can be found in [7] for one loop results, while two loop results are discussed in [50].

We have regarded the mass operator as an insertion and split the Lagrangian into a free piece involving massless fields with the remainder being transported to the interaction Lagrangian. To renormalize the operator, we insert it into a massless Green function, after the fields and couplings have been renormalized in the massless Lagrangian. An attractive feature of the massless field approach is that we can use the MINCER algorithm to perform the actual computations. This algorithm, [51], written in the symbolic manipulation language FORM,
$[52,53]$, is devised to extract the divergences from massless 2 -point functions. The propagators of the massless fields in an arbitrary linear covariant gauge are

$$
\begin{aligned}
\left\langle A_{\mu}^{a}(p) A_{v}^{b}(-p)\right\rangle & =-\frac{\delta^{a b}}{p^{2}}\left[\delta_{\mu \nu}-(1-\alpha) \frac{p_{\mu} p_{v}}{p^{2}}\right], \\
\left\langle c^{a}(p) \bar{c}^{b}(-p)\right\rangle & =\frac{\delta^{a b}}{p^{2}},\langle\psi(p) \bar{\psi}(-p)\rangle=\frac{p^{\prime}}{p^{2}}, \\
\left\langle B_{\mu \nu}^{a}(p) \bar{B}_{\sigma \rho}^{b}(-p)\right\rangle & =-\frac{\delta^{a b}}{2 p^{2}}\left[\delta_{\mu \sigma} \delta_{v \rho}-\delta_{\mu \rho} \delta_{v \sigma}\right], \\
\left\langle G_{\mu \nu}^{a}(p) \bar{G}_{\sigma \rho}^{b}(-p)\right\rangle & =-\frac{\delta^{a b}}{2 p^{2}}\left[\delta_{\mu \sigma} \delta_{v \rho}-\delta_{\mu \rho} \delta_{v \sigma}\right],
\end{aligned}
$$

where $p$ is the momentum. The necessary Feynman diagrams were generated automatically with QGRAF [54].

We first checked that the same two loop anomalous dimensions emerge for the gluon, Faddeev-Popov ghost and quarks in an arbitrary linear covariant gauge as when the extra localizing fields are absent. It was also explicitly verified that the correct coupling constant renormalization constant is found. These results are in agreement with the general argument of the previous subsection.

We have implemented the properties (21) and (22) of the $\lambda^{a b c d}$ coupling in a FORM module, while it was assumed that

$$
\begin{aligned}
\lambda^{\text {acde }} \lambda^{b c d e} & =\frac{1}{N_{A}} \delta^{a b} \lambda^{\text {pqrs }} \lambda^{\text {pqrs }} \\
\lambda^{\text {acde }} \lambda^{b d c e} & =\frac{1}{N_{A}} \delta^{a b} \lambda^{\text {pqrs }} \lambda^{\text {prqs }}
\end{aligned}
$$

which follows from the fact that there is only one rank 2 invariant tensor in a classical Lie group.

At two loops in the $\overline{\mathrm{MS}}$ scheme, we find that

$$
\gamma_{B}(a, \lambda)=\gamma_{G}(a, \lambda)=(\alpha-3) a+\left[\left(\frac{\alpha^{2}}{4}+2 \alpha-\frac{61}{6}\right) C_{A}^{2}+\frac{10}{3} T_{F} N_{f}\right] a^{2}+\frac{1}{128 N_{A}} \lambda^{a b c d} \lambda^{a c b d}
$$

where $N_{A}$ is the dimension of the adjoint representation of the colour group, $a=\frac{g^{2}}{16 \pi^{2}}$ and we have also absorbed a factor of $\frac{1}{4 \pi}$ into $\lambda^{a b c d}$ here and in later anomalous dimensions. These anomalous dimensions are consistent with the general observation that these fields must have the same renormalization constants, in agreement with the output of the Ward identities [7]. A check on (33) is that after the renormalization of the 3-point gluon $B_{\mu v}^{a}$ vertex, the correct gauge parameter independent coupling constant renormalization constant emerges.

We also determined the one loop $\beta$-function for the $\lambda^{a b c d}$ couplings. As this is present in a quartic interaction it means that to deduce its renormalization constant, we need to consider a 4-point function. However, in such a situation the MINCER algorithm is not applicable since two external momenta have to be nullified and this will lead to spurious infrared infinities which could potentially corrupt the renormalization constant. Therefore, for this renormalization only, we have resorted to using a temporary mass regularization introduced into the computation using the algorithm of [55] and implemented in FORM. Consequently, we find the gauge parameter independent anomalous dimension 


$$
\begin{aligned}
\beta_{\lambda}^{a b c d}(a, \lambda)= & {\left[\frac{1}{4}\left(\lambda^{a b p q} \lambda^{c p d q}+\lambda^{a p b q} \lambda^{c d p q}+\lambda^{a p c q} \lambda^{b p d q}+\lambda^{a p d q} \lambda^{b p c q}\right)\right.} \\
& \left.-12 C_{A} \lambda^{a b c d} a+8 C_{A} f^{a b p} f^{c d p} a^{2}+16 C_{A} f^{a d p} f^{b c p} a^{2}+96 d_{A}^{a b c d} a^{2}\right],
\end{aligned}
$$

from both the $\lambda^{a b c d} \bar{B}_{\mu v}^{a} B^{b \mu v} \bar{B}_{\sigma \rho}^{c} B^{d \sigma \rho} \quad$ and $\lambda^{a b c d} \bar{B}_{\mu \nu}^{a} B^{b \mu \nu} \bar{G}_{\sigma \rho}^{c} G^{d \sigma \rho}$ vertices where $d_{A}^{a b c d}$ is the totally symmetric rank four tensor defined by

$$
d_{A}^{a b c d}=\operatorname{Tr}\left(T_{A}^{a} T_{A}^{(b} T_{A}^{c} T_{A}^{d)}\right)
$$

with $T_{A}^{a}$ denoting the group generator in the adjoint representation, [56]. Producing the same expression for both these 4-point functions, aside from the gauge independence, is a strong check on their correctness as well as the correct implementation of the group theory. Moreover, as it should be, $\beta^{a b c d}$ enjoys the same symmetry properties as the tensor $\lambda^{a b c d}$, summarized in (22).

We notice that $\lambda^{a b c d}=0$ is not a fixed point due to the extra

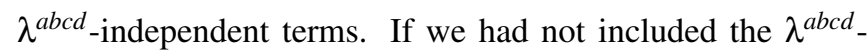
interaction term in the original action, then such a term would inevitably be generated at one loop through quantum corrections, meaning that in this case there would have been a breakdown of renormalizability.

Finally, we turn to the two loop renormalization of the mass $m$. The corresponding operator can be read off from (19) and is given by

$$
\mathcal{M}=\left(B_{\mu v}^{a}-\bar{B}_{\mu v}^{a}\right) F_{\mu \nu}^{a}
$$

We insert $\mathcal{M}$ into a $A_{\mu}^{a}-B_{v \sigma}^{b}$ 2-point function and deduce the appropriate renormalization constant, leading to the $\overline{\mathrm{MS}}$ anomalous dimension

$$
\gamma_{O}(a, \lambda)=-2\left(\frac{2}{3} T_{F} N_{f}-\frac{11}{6} C_{A}\right) a-\left(\frac{4}{3} T_{F} N_{f} C_{A}+4 T_{F} N_{f} C_{F}-\frac{77}{12} C_{A}^{2}\right) a^{2}+\frac{1}{8 N_{A}} f^{a b e} f^{c d e} \lambda^{a d b c} a-\frac{1}{128 N_{A}} \lambda^{a b c d} \lambda^{a b c d}(37)
$$

as the two loop $\overline{\mathrm{MS}}$ anomalous dimension. The gauge parameter independence is again a good check, as the operator $\mathcal{M}$ is gauge invariant.

\section{CONCLUSIONS}

We added a nonlocal mass term (9) to the Yang-Mills action (1), and starting from this, we succeeded in constructing a renormalizable massive gauge model, which is gauge invariant at the classical level and when quantized it enjoys a nilpotent BRST symmetry. This BRST symmetry ensures that the expectation value of gauge invariant operator is gauge parameter independent. We have also proven the equivalence of the massless version of our model with Yang-Mills gauge theories making use of a "supersymmetry" existing between the extra fields in that case. We presented explicit two loop renormalization functions, thereby verifying that the anomalous dimensions of the original Yang-Mills quantities remain unchanged.

Many things could be investigated in the future concerning the gauge model described by (19).

At the perturbative level, it could be investigated which (asymptotic) states belong to a physical subspace of the model, and in addition one should find out whether this physical subspace can be endowed with a positive norm, which would im- ply unitarity. The nilpotent BRST symmetry (24) might be useful for this.

The model (19) is also asymptotically free, implying that at low energies nonperturbative effects, such as confinement, could set in. Proving and understanding the possible confinement mechanism in this model is probably as difficult as for usual Yang-Mills gauge theories.

It would also be interesting to find out whether a dynamically generated term $m(\bar{B}-B) F$ might emerge, which in turn could influence the gluon Green functions. This might also be relevant in the context of gauge invariant $\frac{1}{Q^{2}}$ power corrections, an issue that recently has also attracted attention from the gauge/string duality side, the so-called AdS/QCD [57, 58].

\section{Acknowledgments}

D. Dudal and H. Verschelde are grateful to the organizers of the stimulating IRQCDO6 conference. The Conselho Nacional de Desenvolvimento Científico e Tecnológico (CNPqBrazil), the Faperj, Fundação de Amparo à Pesquisa do Estado do Rio de Janeiro, the SR2-UERJ and the Coordenação de Aperfeiçoamento de Pessoal de Nível Superior (CAPES) are gratefully acknowledged for financial support. 
[1] D. J. Gross and F. Wilczek, Phys. Rev. Lett. 30, 1343 (1973)

[2] H. D. Politzer, Phys. Rev. Lett. 30, 1346 (1973).

[3] M. A. Shifman, A. I. Vainshtein, and V. I. Zakharov, Nucl. Phys. B 147, 385 (1979).

[4] F. V. Gubarev, L. Stodolsky, and V. I. Zakharov, Phys. Rev. Lett. 86, 2220 (2001).

[5] F. V. Gubarev, and V. I. Zakharov, Phys. Lett. B 501, 28 (2001).

[6] M. Lavelle and D. McMullan, Phys. Rept. 279, 1 (1997).

[7] M. A. L. Capri, D. Dudal, J. A. Gracey, V. E. R. Lemes, R. F. Sobreiro, S. P. Sorella, and H. Verschelde, Phys. Rev. D 72, 105016 (2005).

[8] V. N. Gribov, Nucl. Phys. B 139, 1 (1978).

[9] Semenov-Tyan-Shanskii and V.A. Franke, Zapiski Nauchnykh Seminarov Leningradskogo Otdeleniya Matematicheskogo Instituta im. V.A. Steklov AN SSSR, Vol. 120, 159 (1982). English translation: New York: Plenum Press 1986.

[10] D. Zwanziger, Nucl. Phys. B 345, 461 (1990).

[11] G. Dell'Antonio and D. Zwanziger, Nucl. Phys. B 326, 333 (1989).

[12] G. Dell'Antonio and D. Zwanziger, Commun. Math. Phys. 138, 291 (1991).

[13] P. van Baal, Nucl. Phys. B 369, 259 (1992).

[14] D. Dudal, H. Verschelde, and S. P. Sorella, Phys. Lett. B 555, $126(2003)$

[15] J. A. Gracey, Phys. Lett. B 552, 101 (2003).

[16] H. Verschelde, K. Knecht, K. Van Acoleyen, and M. Vanderkelen, Phys. Lett. B 516, 307 (2001).

[17] R. E. Browne and J. A. Gracey, Phys. Lett. B 597, 368 (2004).

[18] J. A. Gracey, Eur. Phys. J. C 39, 61 (2005).

[19] R. E. Browne and J. A. Gracey, JHEP 0311, 029 (2003).

[20] M. J. Lavelle and M. Schaden, Phys. Lett. B 208, 297 (1988).

[21] K. I. Kondo, Phys. Lett. B 514, 335 (2001).

[22] P. Boucaud, A. Le Yaouanc, J. P. Leroy, J. Micheli, O. Pene, and J. Rodriguez-Quintero, Phys. Rev. D 63, 114003 (2001).

[23] P. Boucaud, J. P. Leroy, A. Le Yaouanc, J. Micheli, O. Pene, F. De Soto, A. Donini, H. Moutard, and J. Rodriguez-Quintero, Phys. Rev. D 66, 034504 (2002).

[24] E. Ruiz Arriola, P. O. Bowman, and W. Broniowski, Phys. Rev. D 70, 097505 (2004).

[25] S. Furui and H. Nakajima, hep-lat/0503029.

[26] S. Furui and H. Nakajima, Phys. Rev. D 73, 074503 (2006).

[27] P. Marenzoni, G. Martinelli, and N. Stella, Nucl. Phys. B 455, 339 (1995).

[28] D. B. Leinweber, J. I. Skullerud, A. G. Williams, and C. Parrinello [UKQCD Collaboration], Phys. Rev. D 60, 094507 (1999) [Erratum-ibid. D 61 (2000) 079901].

[29] K. Langfeld, H. Reinhardt, and J. Gattnar, Nucl. Phys. B 621, 131 (2002).

[30] K. Amemiya and H. Suganuma, Phys. Rev. D 60, 114509 (1999).

[31] V. G. Bornyakov, M. N. Chernodub, F. V. Gubarev, S. M. Mo- rozov, and M. I. Polikarpov, Phys. Lett. B 559, 214 (2003).

[32] A. Cucchieri, T. Mendes, and A. R. Taurines, Phys. Rev. D 71, 051902 (2005).

[33] G. Parisi and R. Petronzio, Phys. Lett. B 94, 51 (1980).

[34] J. H. Field, Phys. Rev. D 66, 013013 (2002).

[35] H. Ruegg and M. Ruiz-Altaba, Int. J. Mod. Phys. A 19, 3265 (2004).

[36] H. van Dam and M. J. G. Veltman, Nucl. Phys. B 22, 397 (1970).

[37] R. F. Sobreiro, S. P. Sorella, D. Dudal, and H. Verschelde, Phys. Lett. B 590, 265 (2004).

[38] D. Dudal, R. F. Sobreiro, S. P. Sorella, and H. Verschelde, Phys. Rev. D 72, 01401 (2005).

[39] D. Dudal, J. A. Gracey, V. E. R. Lemes, M. S. Sarandy, R. F. Sobreiro, S. P. Sorella, and H. Verschelde, Phys. Rev. D 70, 114038 (2004).

[40] M. A. L. Capri, V. E. R. Lemes, R. F. Sobreiro, S. P. Sorella, and R. Thibes, Phys. Rev. D 72, 085021 (2005).

[41] D. Dudal, H. Verschelde, V. E. R. Lemes, M. S. Sarandy, R. F. Sobreiro, S. P. Sorella, and J. A. Gracey, Phys. Lett. B 574, 325 (2003).

[42] D. Dudal, H. Verschelde, J. A. Gracey, V. E. R. Lemes, M. S. Sarandy, R. F. Sobreiro, and S. P. Sorella, JHEP 0401, 044 (2004).

[43] R. F. Sobreiro and S. P. Sorella, JHEP 0506, 054 (2005).

[44] D. Dudal, H. Verschelde, V. E. R. Lemes, M. S. Sarandy, S. P. Sorella, and M. Picariello, Annals Phys. 308, 62 (2003).

[45] D. Dudal, H. Verschelde, V. E. R. Lemes, M. S. Sarandy, R. F. Sobreiro, S. P. Sorella, M. Picariello, and J. A. Gracey, Phys. Lett. B 569, 57 (2003).

[46] R. Jackiw and S. Y. Pi, Phys. Lett. B 403, 297 (1997).

[47] D. Zwanziger, Nucl. Phys. B 323, 513 (1989).

[48] D. Zwanziger, Nucl. Phys. B 399, 477 (1993).

[49] O. Piguet and S. P. Sorella, Lect. Notes Phys. M28, 1 (1995).

[50] M. A. L. Capri, D. Dudal, J. A. Gracey, V. E. R. Lemes, R. F. Sobreiro, S. P. Sorella, and H. Verschelde, Phys. Rev. D 74, 045008 (2006).

[51] S. G. Gorishnii, S. A. Larin, L. R. Surguladze, and F. V. Tkachov, Comput. Phys. Commun. 55, 381 (1989).

[52] J. A. M. Vermaseren, math-ph/0010025.

[53] S. A. Larin, F. V. Tkachov, and J. A. M. Vermaseren, The FORM version of MINCER, NIKHEF-H-91-18.

[54] P. Nogueira, J. Comput. Phys. 105, 279 (1993).

[55] K. G. Chetyrkin, M. Misiak, and M. Munz, Nucl. Phys. B 518, 473 (1998).

[56] T. van Ritbergen, A. N. Schellekens, and J. A. M. Vermaseren, Int. J. Mod. Phys. A 14, 41 (1999).

[57] O. Andreev, Phys. Rev. D 73, 107901 (2006).

[58] C. Csaki and M. Reece, hep-ph/0608266. 